\title{
Esophageal Cancer in Esophageal Diverticula Associated with Achalasia
}

\author{
Ah Ran Choi', Nu Ri Chon', Young Hoon Youn', Hyo Chae Paik², Yon Hee Kim³ and Hyojin Park' \\ Departments of ${ }^{1}$ Internal Medicine, ${ }^{2}$ Thoracic Surgery, and ${ }^{3}$ Diagnostic Pathology, Gangnam Severance Hospital, Yonsei University \\ College of Medicine, Seoul, Korea
}

\begin{abstract}
The simultaneous occurrence of achalasia and esophageal diverticula is rare. Here, we report the case of a 68-year-old man with multiple esophageal diverticula associated with achalasia who was later diagnosed with early esophageal cancer. He initially presented with dysphagia and dyspepsia, and injection of botulinum toxin to the lower esophageal sphincter relieved his symptoms. Five years later, however, the patient presented with worsening of symptoms, and esophagogastroduodenoscopy (EGD) was performed. The endoscopic findings showed multifocal lugol-voiding lesions identified as moderate dysplasia. We decided to use photodynamic therapy to treat the multifocal dysplastic lesions. At follow-up EGD 2 months after photodynamic therapy, more lugol-voiding lesions representing a squamous cell carcinoma in situ were found. The patient ultimately underwent surgery for the treatment of recurrent esophageal multifocal neoplasia. After a follow-up period of 3 years, the patient showed a good outcome without symptoms. To manage premalignant lesions such as achalasia with esophageal diverticula, clinicians should be cautious, but have an aggressive approach regarding endoscopic surveillance.
\end{abstract}

Key Words: Esophageal achalasia; Diverticulum; Esophageal; Esophageal; Neoplasms

\section{INTRODUCTION}

The simultaneous occurrence of achalasia and esophageal diverticula is rare. ${ }^{1}$ There have been few reports of esophageal cancer in achalasia and esophageal diverticula. Although most patients with only esophageal diverticula are asymptomatic, patients with both achalasia and esophageal diverticula may present with dysphagia or regurgitation of food. ${ }^{2}$ Additionally, the risk of esophageal cancer is increased in patients with both achalasia and esophageal diverticula. Similar to the pathogenesis of esophageal achalasia, chronic inflammation caused by repeated injury and chronic irritation by stagnated food are thought to result in esophageal carcinogenesis. ${ }^{3,4}$ There have been a few reports of esophageal cancer in achalasia with multiple esophageal diverticula. Our group

\footnotetext{
Received: December 26, 2013 Revised: February 11, 2014

Accepted: March 12, 2014

Correspondence: Hyojin Park

Department of Internal Medicine, Gangnam Severance Hospital, Yonsei University College of Medicine, 211 Eonju-ro, Gangnam-gu, Seoul 135-720, Korea Tel: +82-2-2019-3318, Fax: +82-2-3463-3882, E-mail: HJPARK21@yuhs.ac

(c) This is an Open Access article distributed under the terms of the Creative Commons Attribution Non-Commercial License (http://creativecommons.org/ licenses/by-nc/3.0) which permits unrestricted non-commercial use, distribution, and reproduction in any medium, provided the original work is properly cited.
}

published one such case report in 2009 regarding a case of achalasia associated with multiple large esophageal diverticula. ${ }^{5}$ This is a follow-up of the aforementioned case, in which esophageal carcinoma was subsequently diagnosed in the diverticula that occurred simultaneously with esophageal achalasia.

\section{CASE REPORT}

A 68-year-old man was diagnosed with achalasia associated with multiple esophageal diverticula. He had symptoms of dysphagia and regurgitation of food for 2 years. Thus, barium esophagography, esophageal manometry, and esophagogastroduodenography (EGD) were performed. Barium esophagography showed three right-sided esophageal diverticula along the mid-to-distal esophagus with achalasia (Fig. 1). Initial esophageal manometry showed aperistalsis in the esophageal body, and each swallow resulted in simultaneous contractions (Fig. 2). The average peak pressure of the esophageal body was $56 \mathrm{~mm} \mathrm{Hg}$ as determined by conventional manometry. Therefore, the patient in this case was considered to have vigorous achalasia, defined by an average esophageal body pressure $\geq 37 \mathrm{mmHg}$, rather than the classic form 


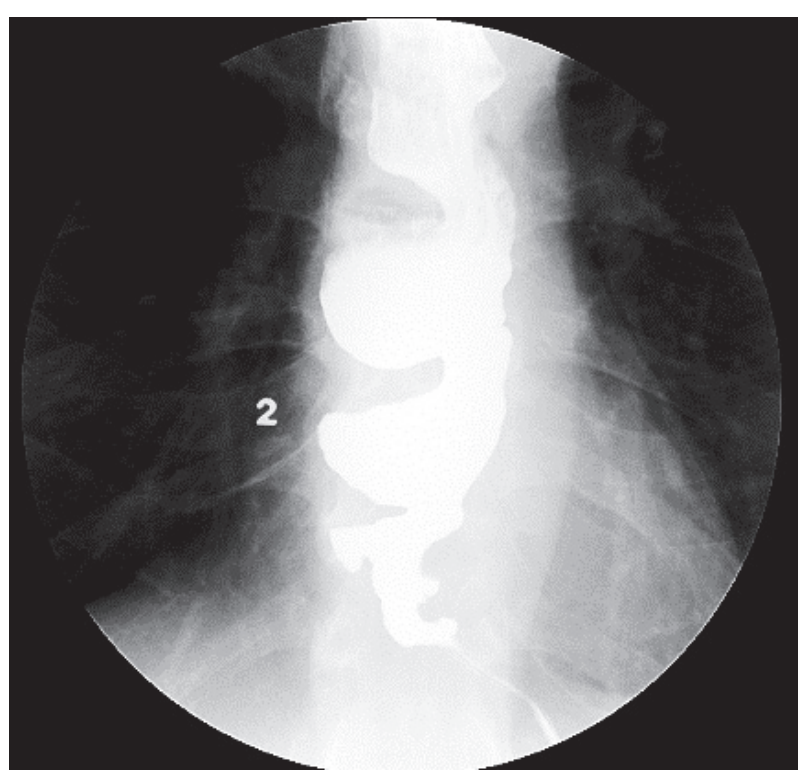

Fig. 1. Barium esophagography. View of a barium esophagogram showing multiple esophageal diverticula and a bird-beak appearance at the esophagogastric junction.

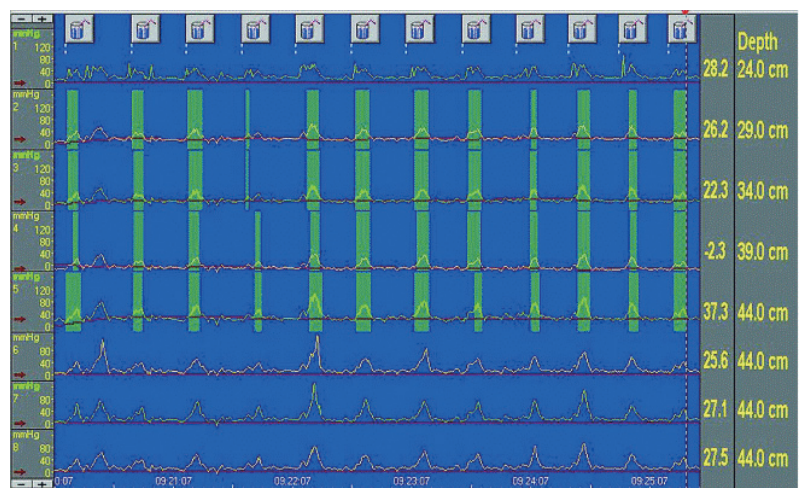

Fig. 2. Esophageal manometry. An esophageal manometric view shows the absence of peristalsis in the esophageal body and simultaneous contractions.

of achalasia associated with lower pressures. ${ }^{6}$ His symptoms improved after botulinum toxin injection. However, dysphagia, regurgitation of food, and epigastric discomfort recurred 5 years later. A follow-up EGD showed a slight increase in the size of the previous diverticula, mild erythematous mucosal changes, and multifocal lugol-voided lesions (Fig. 3). The biopsy result showed a moderate degree of epithelial dysplasia. Because the lesions were located in a large and tortuous diverticulum, performing endoscopic submucosal dissection (ESD) was considered too difficult and dangerous. Photodynamic therapy (PDT) was therefore considered a better option and was administered. Two months after PDT, EGD was repeated to evaluate the response to PDT (Fig. 4). Mucosal nodularity was observed at a nontreated diverticulum, which on biopsy was revealed to be squamous cell carcinoma in situ (Fig. 5). The patient underwent an Ivor-Lewis

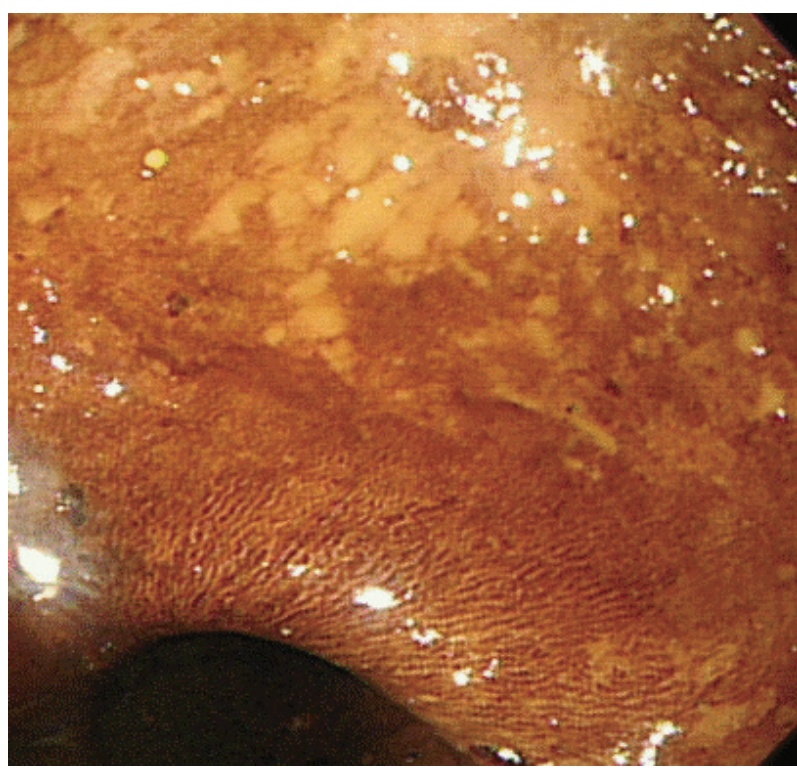

Fig. 3. Esophagogastroduodenography. Endoscopic view at 5 years after botulinum toxin injection therapy. A lugol-voiding lesion is seen at one of the multiple esophageal diverticula.

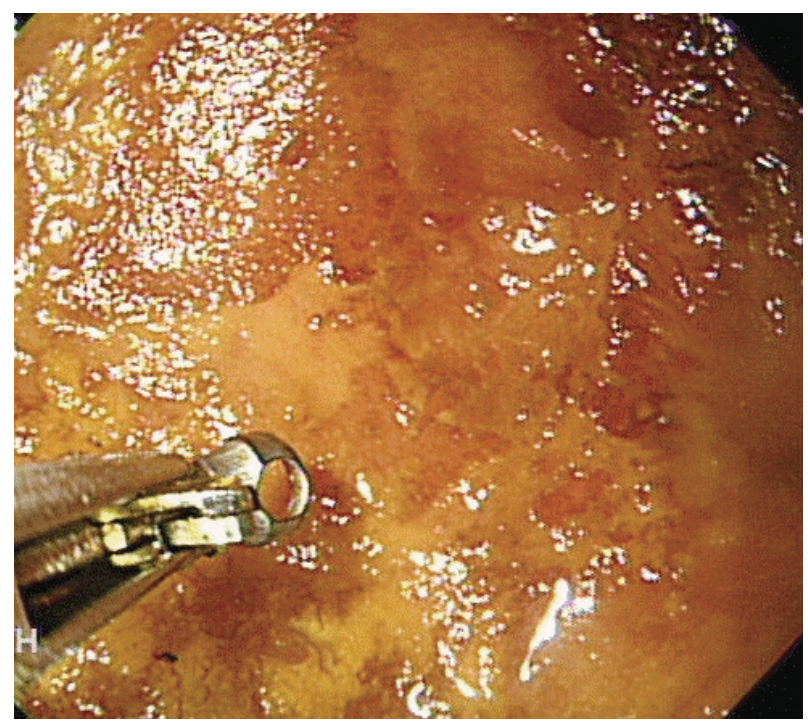

Fig. 4. Esophagogastroduodenoscopy after photodynamic therapy. Endoscopic view 2 months after photodynamic therapy. Another lugol-voiding lesion can be seen. Subsequently, a biopsy was performed.

esophagectomy with regional lymph node dissection for recurrent multifocal esophageal neoplasia, and the final pathologic staging was TisNOM0 (stage 0). The patient showed no evidence of disease at the 3-year follow-up.

\section{DISCUSSION}

Patients with achalasia can develop a variety of complications. Among them, the most serious may be esophageal cancer. The overall prevalence of esophageal squamous cell 


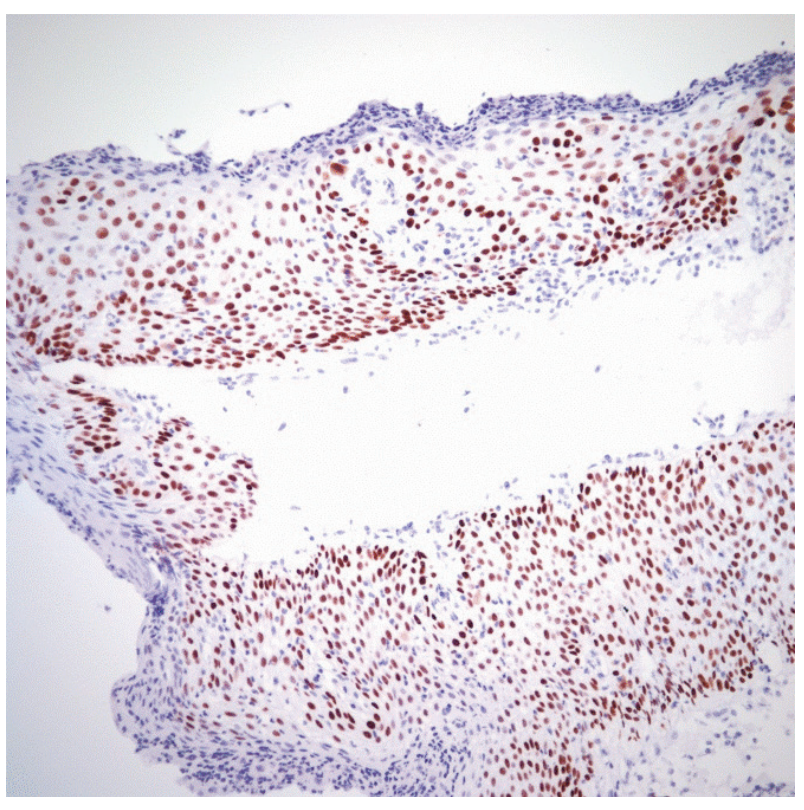

Fig. 5. Histologic findings. A biopsy specimen, from the lesion observed 2 months after photodynamic therapy, shown in Fig. 3 reveals a high-grade epithelial dysplasia and focal squamous cell carcinoma in situ (H\&E stain, $\times 200)$.

carcinoma in patients with achalasia has been estimated to be $3 \%$, accounting for a 50 -fold increase in cancer risk. ${ }^{7}$ In a recent, large prospective cohort study with long-term (mean, 15 years) follow-up data of 448 achalasia patients, the relative hazard ratio for the development of esophageal cancer in achalasia was $28 .^{8}$ Although many cases of esophageal carcinoma in patients with diverticula have been reported, the precise level of cancer risk in esophageal diverticulum remains to be elucidated.

In both achalasia and esophageal diverticulum, swallowed foods stagnate easily. This food and fluid stasis leads to repeated injury of the esophageal epithelium and chronically irritates the mucosal surface. In addition, bacterial overgrowth and impaired clearance of regurgitated gastric acid contents occur during persistent esophageal distention. Due to these diverse factors, chronic inflammation may occur in the esophageal mucosa, which is presumed to develop epithelial hyperplasia, multifocal dysplasia, and finally esophageal cancer.'

Before a diagnosis of cancer, the duration of symptoms is usually at least 15 years in patients with achalasia and at least 10 years in patients with esophageal diverticulum. A large prospective cohort study was conducted with a mean followup period of 15 years after the onset of achalasia symptoms. ${ }^{8}$ Elderly patients have an increased risk of carcinoma. A diverticulum of less than $2 \mathrm{~cm}$ rarely develops into esophageal cancer. $^{10}$

Treatment for esophageal dysplasia or carcinoma arising from achalasia or esophageal diverticulum follows the same principles as esophageal dysplasia or carcinoma without underlying esophageal etiology. Esophageal dysplasia or early esophageal cancer can be treated by endoscopic resection, classic endoscopic mucosal resection (EMR), and ESD, which is widely used. In the present case, however, the diverticula were much too large and tortuous, making EMR or ESD too risky; thus, we performed an operation.

The optimal time and interval for the surveillance of patients with achalasia and/or diverticulum is open-ended. If a patient presents with newly developed symptoms such as weight loss, melena, or hematemesis, endoscopic evaluation should be performed. If not, there is no strong recommendation for endoscopic surveillance. Regarding achalasia, the current version of the American Society of Gastrointestinal Endoscopy guidelines recommends that surveillance should be initiated 15 years after the onset of achalasia symptoms, although the subsequent surveillance interval is not defined. As in the present case, however, patients with both achalasia and diverticula should be considered for endoscopic surveillance earlier than 15 years, as the earlier development of symptoms necessitates shorter surveillance intervals. ${ }^{11}$ Moreover, clinicians should consider chromoendoscopy or narrow-band image endoscopy as well as white light endoscopy for surveillance of these patients.

Although the simultaneous occurrence of achalasia and esophageal diverticula is unusual, this case emphasizes the high risk of esophageal carcinoma arising from both etiologies, as well as the importance of early detection by aggressive endoscopic surveillance.

\section{Conflicts of Interest}

The authors have no financial conflicts of interest.

\section{REFERENCES}

1. Shin MS. Primary carcinoma arising in the epiphrenic esophageal diverticulum. South Med J 1971;64:1022-1024.

2. Sen P, Kumar G, Bhattacharyya AK. Pharyngeal pouch: associations and complications. Eur Arch Otorhinolaryngol 2006;263:463-468.

3. Honda H, Kume K, Tashiro M, et al. Early stage esophageal carcinoma in an epiphrenic diverticulum. Gastrointest Endosc 2003;57:980-982.

4. Kimura H, Konishi K, Tsukioka Y, et al. Superficial esophageal carcinoma arising from the diverticulum of the esophagus. Endoscopy 1997;29:S53-S54.

5. Kim Y, Kim JH, Kim C, Park H. Achalasia associated with multiple esophageal diverticula. Endoscopy 2009;41(Suppl 2):E47-E48.

6. Goldenberg SP, Burrell M, Fette GG, Vos C, Traube M. Classic and vigorous achalasia: a comparison of manometric, radiographic, and clinical findings. Gastroenterology 1991;101:743-748.

7. Dunaway PM, Wong RK. Risk and surveillance intervals for squamous cell carcinoma in achalasia. Gastrointest Endosc Clin N Am 2001;11:425-434.

8. Leeuwenburgh I, Scholten P, Alderliesten J, et al. Long-term esophageal cancer risk in patients with primary achalasia: a prospective study. Am J Gastroenterol 2010;105:2144-2149.

9. Loviscek LF, Cenoz MC, Badaloni AE, Agarinakazato O. Early cancer 
in achalasia. Dis Esophagus 1998;11:239-247.

10. Herbella FA, Dubecz A, Patti MG. Esophageal diverticula and cancer. Dis Esophagus 2012;25:153-158.
11. Hirota WK, Zuckerman MJ, Adler DG, et al. ASGE guideline: the role of endoscopy in the surveillance of premalignant conditions of the upper GI tract. Gastrointest Endosc 2006;63:570-580. 